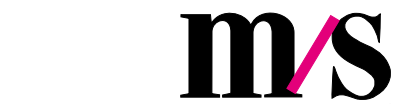

médecine/sciences $1999 ; 15: 307-9$

\section{Jean-Jacques Mercadier}

\title{
PHYSIOLOGIE EN CARDIOLOGIE, UN RETOUR NÉCESSAIRE MAIS MENACÉ
}

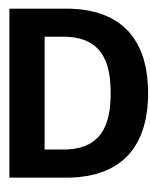
ans son éditorial du précédent numéro que médecine/sciences consacrait, en octobre dernier, au cœur, mon ami Bernard Swynghedauw déplorait, à juste titre, une certaine faiblesse actuelle de la physiologie (et en corollaire de la physiopathologie) dite d'organe [1]. Il n'allait pas jusqu'à localiser cette faiblesse dans notre pays ou même en Europe, mais sa référence à une initiative de l'American Heart Association en faveur de l'intégration des divers aspects de la biologie cardiaque allait malgré tout en ce sens, comme si on avait déjà, là-bas, pris conscience d'un problème.

Il faut reconnaitre que le sommaire des deux numéros " coeur » successifs, avec un premier numéro très centré sur le développement et la génétique des cardiomyopathies a accentué, d'une manière involontairement provocatrice, cette prévalence qui semble être dévolue actuellement à la génétique par rapport à la physiologie d'organe ou de "système » (on parlait autrefois aussi d'«appareil»). Cette distinction me semble artificielle pour au moins deux raisons. La première est que l'époque de l'identification des principales mutations des cardiopathies monogéniques est déjà révolue. Nous en sommes au stade de l'élucidation des mécanismes pathogènes de ces mutations au moyen d'études utilisant presque toujours des animaux transgéniques, études qui sont tenues de prendre en compte la dimension de la physiologie d'organe, voire de système. La découverte de mutations dans de très importantes protéines du myocyte cardiaque, outre les retombées directes qu'elles ont eues pour la compréhension du fonctionnement de la molécule ou du complexe moléculaire concerné, a créé de fait le besoin d'une physiopathologie « inverse » qui doit maintenant expliquer, à travers une continuité de mécanismes pathologiques à l'échelon moléculaire, intermoléculaire, subcellulaire, cellulaire, tissulaire, de l'organe puis du système, comment telle mutation de la chaîne lourde $\beta$ de la myosine par exemple, aboutit à un ventricule hypertrophié [2]. Est-il vraiment nécessaire de chercher à déterminer le poids relatif exact de la physiologie d'organe dans ce continuum de connaissances qu'il faut maintenant réunir dans des délais très courts pour avoir une chance de publier au plus haut niveau?

La seconde raison est que, si la physiologie est bien la science des fonctions du vivant, alors elle recoupe tous les niveaux de connaissance auxquels les techniques du moment nous donnent la possibilité d'accéder : relation structure-fonction d'une molécule, manière dont les molécules s'associent pour remplir une fonction dans la cellule, rôle du niveau subcellulaire d'organisation des complexes moléculaires dans l'accomplissement d'une fonction de la cellule... avec en miroir l'étude de l'infinité des dysfonctionnements qui peuvent résulter, à chaque niveau, d'une mutation, d'une modification quantitative, d'une désorganisation de la micro-architecture cellulaire. Les articles des deux numéros «cœur » illustrent de manière frappante ce va-et-vient nécessairement permanent entre la molécule et 
l'organe et même au-delà : from bench to bedside disent nos collègues anglosaxons! La distance de la paillasse au lit du malade est souvent infime. Dans le présent numéro, Sylvain Richard et al. (p.329 de ce numéro) montrent bien combien ténue est la distance conceptuelle entre un mécanisme moléculaire, le ralentissement de la cinétique d'inactivation du courant calcique des myocytes, et un phénomène observé au niveau de l'organe : la positivité de la relation entre la force de la contraction et la fréquence cardiaque, et comment une altération encore mal comprise de ce mécanisme participe à la disparition de cette relation dans le coeur défaillant. Stéphane Hatem et al. (p. 338 de ce numéro) décrivent le lieu et des acteurs possibles de cette altération au niveau de la «synapse calcique ", alcôve qui héberge les ébats du couple canal calcique - récepteur de la ryanodine et les nombreux partenaires aux noms plus ou moins exotiques dont ils semblent ne pouvoir se passer : sorcine, jonctine, triadine, FKBP12... Tout un univers de relations à découvrir.

Tout aussi étroite la distance entre la fonction des molécules et la contraction du cœur dans des conditions normales ou pathologiques dans les articles de Jean-Luc Balligand et al. et de Bertrand Crozatier et Claude Delcayre (p. 310 et p. 345 de ce numéro). Ce dernier article révèle comment un mécanisme de pure physiologie «d'organe » s'il en est, la « loi de Starling » qui permet au cœur d'augmenter sa force de contraction mais cette fois lors de l'étirement de ses myocytes, est simultanément à l'origine d'un des principaux processus d'adaptation de ces cellules : leur hypertrophie. Cellules qui savent également se défendre contre des épisodes répétés d'ischémie comme l'indique Michel Ovize avec, dans les deux cas, un rôle probablement central de la protéine-kinase $\mathrm{C}$. Le mécanisme de défense contre l'acidose décrit par Danielle Feuvray et Morris Karmazyn (p.322 de ce numéro), est en revanche, plus directement délétère pour le myocyte. De même, les altérations des courants ioniques membranaires lors des cardiopathies décrits par Alain Coulombe et al. (p.359 de ce décrits par Pascale Guicheney et al. dans le premier numéro [3], au moins en partie responsables des arythmies qui constituent finalement le mode de décès le plus fréquent de la plupart des cardiopathies. L'article de Frédérique Tesson et al. (p. 369 de ce numéro) vient à point conclure ces deux séries de synthèses en ouvrant la perspective des cardiopathies multigéniques à forte composante environnementale. Même si de rares formes de cardiomyopathies dilatées ont une origine monogénique, l'exemple de la cardiomyopathie du hamster syrien montre bien comment une mutation dans le gène codant pour la $\delta$-sarcoglycane, protéine du complexe d'ancrage de la dystrophine dans le sarcolemme, peut tuer probablement d'un trouble du rythme, à travers une longue histoire d'hypertrophie et/ou de dilatation (selon les lignées) et d'altérations électrophysiologiques diverses [4]. Du pain sur la planche pour les physio... pathologistes, apporté par les généticiens ! Comme le disait Alain Prochiantz dans ces mêmes colonnes en novembre, la génétique est bien la chance de la physiologie [5]. On pourrait dire tout aussi bien que la physiologie (et les souris transgéniques!) est la chance de la génétique.

A travers ce panorama, il est clair qu'il n'existe pas de solution de continuité de la physiologie moléculaire à la physiopathologie d'organe ou de système, de la génétique au phénotype et inversement, et que notre communauté scientifique et médicale est présente dans l'ensemble des champs thématiques de la physiologie et de la physiopathologie cardiaques. Mon inquiétude est plutôt dans les moyens dont elle dispose. Je faisais part il y a un an aux lecteurs de médecine/sciences [6] de mon enthousiasme devant le travail de Gomez et al. [7] avançant une nouvelle hypothèse pour expliquer le dysfonctionnement contractile du myocarde hypertrophié et défaillant. Anna-Maria Gomez a pu réaliser ce travail de mesure des concentrations du calcium libre dans des domaines subcellulaires du myocyte chez John Lederer à Baltimore (MA, USA), grâce à une installation de microscopie confocale à haute résolution temporelle et spatiale en permanente évolution. Je ne suis pas certain qu'elle a eu l'occasion d'utiliser un tel outil depuis les dix-huit mois qu'elle est en France. Sans entrer dans les détails, comment les deux ou trois progrès majeurs de la cardiologie fondamentale en 1998 auraient-ils pu être réalisées en France ou même en Europe à la vitesse à laquelle ils l'ont été outre-Atlantique? Je pense par exemple à la découverte d'une nouvelle voie de signalisation de l'hypertrophie du myocyte, liée à la calcineurine, phosphatase inhibée par la ciclosporine A. La calcineurine permet la translocation nucléaire de NFAT3 et ainsi l'hypertrophie des myocytes in vitro [8] et in vivo dans certaines cardiopathies hypertrophiques réalisées chez la souris qui sont prévenues par un traitement par la ciclosporine [9]. Je pense aussi à la démonstration du rôle de Goq dans l'hypertrophie d'origine hémodynamique par réalisation d'une surcharge de pression $\mathrm{du}$ ventricule gauche chez des souris chez lesquelles cette protéine a été fonctionnellement inactivée par transgenèse. Ces souris développent moins d'hypertrophie que les souris témoins [10]. Au contraire, d'autres souris au ventricule hypertrophié par surexpression de cette même protéine développent une insuffisance cardiaque quand elles sont soumises à la surcharge de pression [11]. Ces études ont été réalisées, pour l'essentiel, à Cincinnati, ville moyenne du centre des États-Unis où l'on produit des souris transgéniques à peu près comme nous faisons des PCR. Il est vrai que la transgenèse en cardiologie revêt souvent, outre-Atlantique, un aspect peu imaginatif, un côté un peu trop criblage systématique qui nous fait sourire, nous qui avons des idées ! $\mathrm{Au}$ moins cette approche offre-t-elle l'avantage de pouvoir tester rapidement l'implication de nombreuses protéines dans des processus physiopathologiques variés. Quoi qu'il en soit, ne pas considérer qu'une différence d'un facteur 10 (si ce n'est 100) dans les possibilités de réalisation et d'exploration des animaux transgéniques entre les États-Unis et notre pays (je ne parle que du domaine de la recherche cardiovasculaire bien sûr !) constitue un problème majeur risque de nous conduire très rapide- 
ment à un retard qu'il sera difficile de rattraper. Le développement des IFR en France et les nouvelles perspectives européennes, en particulier celles offertes par le $5^{\mathrm{e}}$ Programme cadre européen de recherche et de développement (PCRD), permettront-ils de combler rapidement ce fossé et constitueront-ils les conditions suffisantes pour faire basculer complètement notre recherche cardiovasculaire vers le $\mathrm{XXI}^{\mathrm{e}}$ siècle ? Les numéros «cœur» de médecine/sciences des années 2000 nous le diront.

Je ne voudrais pas terminer sans avoir une pensée pour Édouard Corabœuf, grand électro... physiologiste qui nous a quittés en septembre. Au moins la moitié des auteurs de ces deux numéros ont été ses élèves et il a largement participé à la rédaction de l'article d'Alain Coulombe et al., en particulier en dessinant de sa main, du trait sûr et précis que ses proches lui connaissaient, chacune des figures de l'article. Son ami Edward Carmeliet, lui rend hommage dans ce même numéro. A titre personnel, je tiens aussi à remercier la Rédaction de médecine/sciences d'avoir donné l'occasion à notre communauté de s'exprimer ainsi dans le journal. Je remercie enfin tous les auteurs qui ont répondu présent en acceptant le défi d'écrire de véritables articles de synthèse, authentiquement originaux, quand les contraintes de la compétition internationale poussent souvent à délaisser ce genre d'exercice

\section{RÉFÉRENCES}

1. Swynghedauw B. Génétique en cardiologie, un evénement inattendu. Med Sci 1998 ; 14 : 1007-8.

2. Bonne G, Carrier L, Schwartz K. Vers une meilleure compréhension du rôle de la protéine C dans la contraction cardiaque. Med Sci 1995 ; 11 : 1739-41.

3. Guicheney, Barhanin J, Le Marec H. Bases moléculaires des arythmies héréditaires. Med Sci 1998 ; 14 : 1025-35.

4. Thuringer D, Deroubaix E, Coulombe A, Corabœuf E, Mercadier IJ. Ionic basis of the action potential prolongation in ventricular myocytes from Syrian hamsters with dilated cardiomyopathy. Cardiovasc Res 1996 ; 31 : $747-57$.
5. Prochiantz A. Biologie des mauvaises humeurs. Med Sci 1998 ; 14 : 1155-6.

6. Mercadier J, Hatem S. Recaptage ou relargage? ou les caprices de la signalisation calcique du myocyte dans l'hypertrophie et l'insuffisance cardiaques. Med Sci 1997 ; 13 : 1454-8.

7. Gomez AM, Valdivia HH, Cheng $\mathrm{H}$, et al. Defective excitation-contraction coupling in experimental cardiac hypertrophy and heart failure. Science 1997 ; 276 : 800-6.

8. Molkentin JD, Lu JR, Antos CL, Markham B, Richardson J, Robbins J, Grant SR, Olson EN. A calcineurin-dependent transcriptional pathway for cardiac hypertrophy. Cell 1998 ; $93: 215-28$

9. Sussman MA, Lim HW, Gude N, et al. Prevention of cardiac hypertrophy in mice by calcineurin inhibition. Science 1998 ; 281 : 1690-3.

10. Akhter SA, Luttrell LM, Rockman HA, Iaccarino G, Lefkowitz RJ, Koch WJ. Targeting the receptor-Gq interface to inhibit in vivo pressure overload myocardial hypertrophy. Science 1998 ; 280 : 574-7.

11. Sakata Y, Hoit BD, Liggett SB, Walsh RA, Dorn GW 2nd. Decompensation of pressure-overload hypertrophy in Gaq-overexpressing mice. Circulation $1998 ; 97$ : 1488-95.

\section{Bonne chance, Élisabeth !}

Le Docteur Élisabeth Bursaux, qui avait rejoint médecine/sciences depuis six ans, et y assumait les fonctions de Rédacteur en chef-adjointe, responsable de la partie magazine, a quitté ses fonctions le $1^{\mathrm{er}}$ mars. Son départ est pour nous une grande tristesse, car elle avait su apporter à médecine/sciences toute sa compétence et l'enthousiasme - voire l'acharnement - que tous les chercheurs, biologistes et cliniciens, qu'elle a amenés à enrichir le magazine de $\mathrm{m} / \mathrm{s}$ ont pu tant de fois apprécier. Ce départ est aussi pour nous, toutefois, l'occasion de grandes joies. Pour Élisabeth d'abord, qui entre aujourd'hui par la grande porte dans le plus grand journal du soir français où elle va pouvoir défendre, encore et toujours, la science et le monde de la recherche scientifique pour lesquels elle s'est toujours totalement engagée, comme chercheur, directeur de recherche à l'Inserm d'abord, comme responsable de médecine/sciences ensuite. C'est une joie pour toute l'équipe de médecine/sciences, ensuite, car nous prenons comme un grand honneur le geste de la rédaction de ce grand média qui est venue chercher dans notre revue la qualité scientifique et la compétence journalistique.

Les comités éditoriaux de médecine/sciences, à Paris et à Montréal, auront fort à faire pour assumer les tâches qu'Élisabeth remplissait jusque-là. Le Docteur Laure Coulombel a accepté de prendre celle de responsable scientifique de la partie magazine, et le Docteur François Flori celle de rédacteur en chefadjoint. Ils seront bientôt rejoints par un(e) assistant(e) du rédacteur en chef, poste pour lequel nous venons de lancer un appel d'offres grâce à l'appui de l'Inserm qui le met à notre disposition.

Bonne chance, Élisabeth, dans tes nouvelles attributions, et merci au nom des membres des Comités de médecine/sciences, et de tous ses lecteurs, pour l'extraordinaire travail que tu as accompli pour notre revue!

Marc Peschanski

Rédacteur en chef, Paris 